\title{
Changes of memory B-and T-cell subsets in lupus nephritis patients
}

\author{
Joanna Kosalka*, Bogdan Jakiela*, Jacek Musial \\ Jagiellonian University Medical College, Department of Medicine, Krakow, Poland \\ *Both authors have equally contributed to this manuscript
}

\begin{abstract}
Introduction. Renal involvement in systemic lupus erythematosus (SLE) is associated with production of antibodies to double stranded DNA, deposition of immune complexes and organ damage. These processes have been linked with abnormalities in B- and T-cell memory compartments. The aim of the study was to analyze subsets of peripheral memory B-cells and T-cells in lupus nephritis (LN) patients.

Material and methods. We used multicolor flow cytometry to analyze major memory subsets of peripheral blood B-cells (defined by CD27, IgD and CD21) and T-cells (CD45RA, CD45RO, CCR7) in 32 patients with active or inactive $\mathrm{LN}$, and 23 control subjects.

Results. Lupus nephritis patients were characterized by increased percentage of immature/early-transitional B-cells (CD27-IgD+CD21-), higher frequency of activated switched memory (SM, CD27+IgD-CD21-) and exhausted memory B-cells (CD27-IgD-), and decrease in non-switched memory (NSM, CD27+IgD+) B-cells. $\mathrm{CD} 21^{\text {low }}$ subsets (immature and activated B-cells) were particularly expanded in patients with active disease. In both groups of $\mathrm{LN}$ patients we observed decline in the absolute count of NSM B-cells. It was paralleled by lymphopenia in naïve CD4+ T-cell compartment and increase in the frequency of effector memory T-cells, and these changes were more pronounced in active $\mathrm{LN}$.

Conclusions. B-cell memory compartment in LN is deficient in NSM cells and during active disease it is further skewed towards SM and exhausted memory phenotypes, most likely as a cause of chronic antigenic stimulation. Parallel changes in T-helper cell subsets suggest a similar mechanism of SLE-related lymphopenia for both B-cell and T-cell compartment. (Folia Histochem Cytobiol. 2016, Vol. 54, No. 1, 32-41)
\end{abstract}

Key words: systemic lupus erythematosus; lupus nephritis; immune memory; B-cells; T-cells; flow cytometry

\section{Introduction}

Systemic lupus erythematosus (SLE) is a complex autoimmune disorder with B-cell hyper-reactivity, immune complex deposition, and organ damage. Lupus nephritis (LN) develops in $~ 50 \%$ SLE patients, representing the main cause of morbidity and mortality $[1,2]$. The significance of self-reacting B-cells in the pathogenesis of $\mathrm{LN}$ is supported by a strong causal link between autoantibodies against double stranded DNA (dsDNA) and renal involvement [3, 4]. Addi-

\footnotetext{
Correspondence address: J. Musial, M.D., Ph.D.

Jagiellonian University Medical College

Department of Medicine

Skawinska 8, 31-066 Krakow, Poland

e-mail: mmmusia@cyf-kr.edu.pl
}

tionally, rise in the titer of anti-dsDNA antibodies occurs years before the clinical onset of proliferative LN $[5,6]$. It has been suggested that autoimmune inflammation results from repeated stimulation of autoreactive memory T- and B-cells, and subsequent effector responses mediated in part by circulating autoantibodies.

During primary immune response to T-cell dependent (TD) antigens naïve B-cells receive signals from T-helper cells and proliferate rapidly forming germinal centers (GC) in lymphoid tissues. Many of responding B-cells undergo somatic hypermutation and class switching [7]. Memory B-cells generated during GC reaction can be recognized by surface expression of $\mathrm{CD} 27$, and are classified as switched memory cells (SM; CD27+IgD-), or non-switched (i.e. $\mathrm{IgM}+)$ memory cells $(\mathrm{NSM} ; \mathrm{CD} 27+\mathrm{IgD}+)$ [8]. 
During secondary antigen exposure SM B-cells proliferate rapidly and differentiate into antibody secreting cells, while NSM cells are able to reinitiate GC reaction and replenish SM fraction $[9,10]$. Because B-cell response to nuclear auto-antigens requires T-cell help, formation of pathogenic autoantibodies in $\operatorname{SLE}$ (e.g. anti-dsDNA) follows the same rules [11]. As a consequence, in all SLE patients a pool of autoreactive memory B- and T-cells is steadily generated. It has been hypothesized that continuous presence to autoantigens favors differentiation of effector memory (EM) T-cells, which mount a pathogenic inflammatory response upon re-exposure to self-antigens [12]. Similarly to other memory cells, they are long-lived and difficult to eliminate, thus pose a considerable therapeutic challenge [13].

Lymphocyte phenotyping in SLE patients revealed expansion of $\mathrm{CD} 27+$ memory B-cells with parallel decline in naïve compartment, but little is known if they are intrinsic or develop secondarily to disease-related inflammation [14-16]. Interestingly, memory B-cells in lupus patients often display 'double negative' (i.e. CD27-IgD-) phenotype and correlate with renal involvement $[17,18]$. Additionally, several groups reported on marked decline in the frequency of CD27+ IgD + B-cells (NSM) mostly in patients with active disease [18-20]. B-cell lymphopenia is another typical feature of SLE, but less is known if it affects all B-cell subsets, and whether it is associated with similar abnormalities in T-cell compartment. Therefore in this study we aimed to analyze subsets of circulating B-cells in LN patients, in search for potential markers associated with disease activity. Additionally, we compared changes in B-cell and T-cell subsets depending on SLE-related lymphopenia.

\section{Material and methods}

Characteristics of the patients. Blood samples were taken from 34 systemic lupus erythematosus (SLE) patients with renal involvement, and 23 control subjects (apparently healthy, with negative laboratory tests for antinuclear and antiphospholipid autoantibodies). SLE was diagnosed based on updated criteria of the American College of Rheumatology [21]. LN was confirmed by renal biopsy and classified according to International Society of Nephrology/Renal Pathology Society (ISN/RPS) criteria [22] as class II (3\%), III (15\%), IV $(56 \%)$ or V $(8 \%)$. In 6 patients $(18 \%) \mathrm{LN}$ was diagnosed based on overt renal symptoms (proteinuria, active urinary sediment) during lupus flare. We enrolled $16 \mathrm{LN}$ patients with disease exacerbation (active-LN, SLE Disease Activity Index [SLEDAI] $>6$, urine protein $>1 \mathrm{~g} /$ day or $>2$-fold increase with active urinary sediment) and 18 patients in remission (inactive-LN, SLEDAI $\leq 6$, urine protein $<1$ and normal urinary sediment) [23]. All but one active-LN patients had additional SLE symptoms: hematologic $(88 \%$, mostly lymphopenia), joint symptoms (50\%), skin lesions (50\%), and pleuritis (19\%). LN patients were not treated with biological agents targeting B-cells (e.g. rituximab). Additionally, they were not treated with cyclophosphamide in the preceding 6 months. The clinical and laboratory characteristics of LN patients are summarized in Table 1. The study was approved by the Ethics Committee of the Jagiellonian University and informed written consent was obtained from all participants.

Flow cytometry. Aliquots of EDTA anticoagulated blood were stained with a mixture of monoclonal antibodies (all from BD Biosciences, Franklin Lakes, NJ, USA) for detection of CD19+ B-cell (anti-CD45, CD3, CD19, CD21, CD27, IgD; gating strategy shown in Figures 1A, B) and T-cell memory subpopulations (anti-CD45, CD3, CD4, CD8, CD45RA, CD45RO, CCR7). Samples were fixed with FACS lysing solution (BD Biosciences), washed and analyzed in FACS Canto II flow cytometer (BD Biosciences). Based on differential expression of surface markers the following B-cell subsets were identified: immature/ /early-transitional (I/T, CD27-IgD+CD21 $\left.{ }^{\text {low }}\right)$, late-transitional/naïve (CD27-IgD+CD21+), non-switched memory (NSM, CD27+IgD+), resting class-switched memory (SM, $\mathrm{CD} 27+\mathrm{IgD}-\mathrm{CD} 21+)$, activated SM (CD27+IgD-CD21 $\left.{ }^{\text {low }}\right)$, and double-negative (CD27-IgD-) exhausted memory (ExM) as summarized in Figure 1C [8, 16, 23, 25]. Using T-cell panel we identified four subsets within CD4+ or CD8+ gate: naïve (CD45RA+CD45RO-CCR7+), central memory $\left(\mathrm{T}_{\mathrm{CM}}, \mathrm{CD} 45 \mathrm{RA}-\mathrm{CD} 45 \mathrm{RO}+\mathrm{CCR} 7+\right)$, effector memory ( $\left.\mathrm{T}_{\mathrm{EM}}, \mathrm{CD} 45 \mathrm{RA}-\mathrm{CD} 45 \mathrm{RO}+\mathrm{CCR} 7-\right)$, and effector memory $\mathrm{CD} 45 \mathrm{RA}+\left(\mathrm{T}_{\mathrm{EMRA}}, \mathrm{CD} 45 \mathrm{RA}+\mathrm{CD} 45 \mathrm{RO}-\mathrm{CCR} 7-\right)$ [26, 27].

Statistical methods. Statistical analysis was performed using GraphPad Prism 5.0 (GraphPad Software, Inc., San Diego, CA, USA). Data is presented as medians and 25-75 quartile ranges if not otherwise stated. Differences between two groups were determined by a Mann-Whitney U test. Differences between at least three groups were determined by ANOVA or Kruskal-Wallis test if justified. Spearman's rank sum test was used to analyze correlation coefficients. $P$ value $<0.05$ was considered significant.

\section{Results}

\section{Decreased frequency of non-switched memory \\ $B$-cells and signs of B-cell exhaustion in $L N$}

The phenotypic characteristics of the analyzed B-cell subsets have been shown in Figure 1C. There was a large variability in the percentage of CD19+ B-cells in LN patients, with no difference in comparison with control group. B-cell counts were significantly lower in $\mathrm{LN}$ patients both with active and inactive disease 
Table 1. Characteristics of the subjects studied

\begin{tabular}{|c|c|c|c|}
\hline & Active LN $(n=16)$ & Inactive LN (n = 18) & Control group $(n=23)$ \\
\hline Age (years) & $35[30-47]$ & $35[29-50]$ & 34 [27-41] \\
\hline Sex (Females, \%) & $12(75 \%)$ & $15(83 \%)$ & $18(78 \%)$ \\
\hline SLE duration (years) & $10[3-14.5]$ & $9.5[4-15]$ & n.a. \\
\hline Symptoms (in history) & - & - & n.a. \\
\hline Skin & $15(94 \%)$ & $14[78 \%]$ & \\
\hline Joints & $16(100 \%)$ & $15[83 \%]$ & \\
\hline Pleuropericarditis & $6(38 \%)$ & $8[44 \%]$ & \\
\hline Neurologic & $1(6 \%)$ & $2[11 \%]$ & \\
\hline Hematologic & $16(100 \%)$ & $16[89 \%]$ & \\
\hline LN class II/III/IV/V(unknown) ${ }^{1}$ & $1 / 2 / 9 / 2(2)$ & $0 / 3 / 10 / 1(4)$ & n.a. \\
\hline SLEDAI (score) & $21.5[14-24]^{\# \#}$ & $2[0-4]$ & n.a. \\
\hline ANA (titer) & 10240 [5120-20480] & 5120 [2240-10240] & none \\
\hline Anti-dsDNA (titer) & $480[160-2560]^{\# \#}$ & $40[<10-160]$ & none \\
\hline Proteinuria (g/day) & $2.4[1.7-5.2]^{\# \#}$ & $0.1[0.06-0.39]$ & n.a. \\
\hline Creatinine (serum, $\mu \mathrm{mol} / \mathrm{L}$ ) & 69.5 [65.2-91.7] & $66[60.2-80.5]$ & $71[65-79]$ \\
\hline $\mathrm{C} 3 \mathrm{c}(\mathrm{mg} / \mathrm{dL})$ & $0.545[0.44-0.785]^{* *, \# \#}$ & $1.035[0.790-1.185]^{*}$ & $1.15[0.963-1.41]$ \\
\hline $\mathrm{CRP}(\mathrm{mg} / \mathrm{L})$ & $17.1[9.1-25.8]^{* *, \# \#}$ & $1.7[0.6-7.7]^{*}$ & $0.7[0.3-2.8]$ \\
\hline $\mathrm{WBC}\left(\cdot 10^{3} / \mathrm{uL}\right)$ & $5.2[4.1-8.1]$ & $6.4[4.4-8.8]$ & $6.3[5.7-7.9]$ \\
\hline Lymphocytes $\left(\cdot 10^{3} / \mathrm{uL}\right)$ & $0.67[0.37-1.26]^{* *}$ & $0.89[0.65-1.38]^{* *}$ & $1.82[1.61-2.21]$ \\
\hline Current systemic GCS (mg/d) & $8[4-16]^{\#}$ & $4[0-4]$ & none \\
\hline Cumulative CTX dose (g) & $0[0-8.6]^{\# \#}$ & $9.5[6.1-26.2]$ & n.a. \\
\hline
\end{tabular}

${ }^{1}$ According to International Society of Nephrology/Renal Pathology Society criteria [22]. Data are presented as medians [25-75 quartiles]. ${ }^{* * *} P<0.05$ and ${ }^{*} P<0.01$, respectively, in comparison with control group; $\#,{ }^{* \#} P<0.05$ and $P<0.01$, respectively, in comparison with Inactive LN. Abbreviations: ANA — anti-nuclear antibodies; C3c — complement component C3c; CRP - C-reactive protein; CTX — cyclophosphamide; GCS - glucocorticoids (recounted for methylprednisolone); LN — lupus nephritis; SLEDAI — SLE disease activity index; WBC — white blood cells

(Figure 1D). LN patients showed high variability in the distribution of main B-cell subsets, which was more evident in patients with the active disease (Figure 1E). The percentage of early-transitional B-cells $\left(\mathrm{CD} 27-\mathrm{IgD}+\mathrm{CD} 21^{\text {low }}\right)$ was increased in the active $\mathrm{LN}$ (median $6.5 \%$ of $\mathrm{CD} 19+$ ) as compared with inactive $\mathrm{LN}(3.0 \%, P<0.05)$ and control $(2.1 \%$, $P<0.01$ ) groups (Figure 1F). In all patients with active $\mathrm{LN}$, and in majority $(\mathrm{n}=15,83 \%)$ of patients with inactive disease, we observed a marked decrease (median $2.4 \%$ and $2.3 \%$, respectively) in the percentage of NSM B-cells (Figure 1E, F) as compared with control subjects $(13.4 \%)$. This was a characteristic feature of B-cell memory compartment, distinctive to SLE (Figure 1G). Additionally, we observed increased frequency of activated SM cells in active LN (median $12.6 \%$ ) as compared with other groups (inactive $\mathrm{LN}$ $-6.0 \%, P<0.05$, control $-2.5 \%, P<0,01)$.

Moreover, B-cell compartment in LN patients had signs of memory exhaustion, as evidenced (Figure 1E,F) by increased percentage of double negative (CD27-IgD-) CD19+ B-cells (median 6.8\% in active- and 9.9\% in inactive-LN vs. $2.4 \%$ in control group, $P<0.01$ ). Next, we compared absolute counts of B-cells in order to identify changes associated with SLE-induced lymphopenia. As shown in Figure $1 \mathrm{H}$, there was a strong ( $>10$-fold) decrease in the number of NSM B-cells in LN patients (independently of the activity of the disease) as compared with control group, but we also found a significant, though less pronounced decrease in the count of naïve and resting SM B-cells.

\section{Subsets of CD21 ${ }^{\text {low }} B$-cells correlate with $L N$ activity} Overall, there was a strong association of B-cells with decreased expression of CD21 (comprised of early-transitional and activated/exhausted B-cells) with the activity of the disease (Figure 2A). In particular, we showed a correlation (Figure 2B, C) between the percentage of immature/early-transitional B-cells and various markers of SLE activity, e.g. SLEDAI 


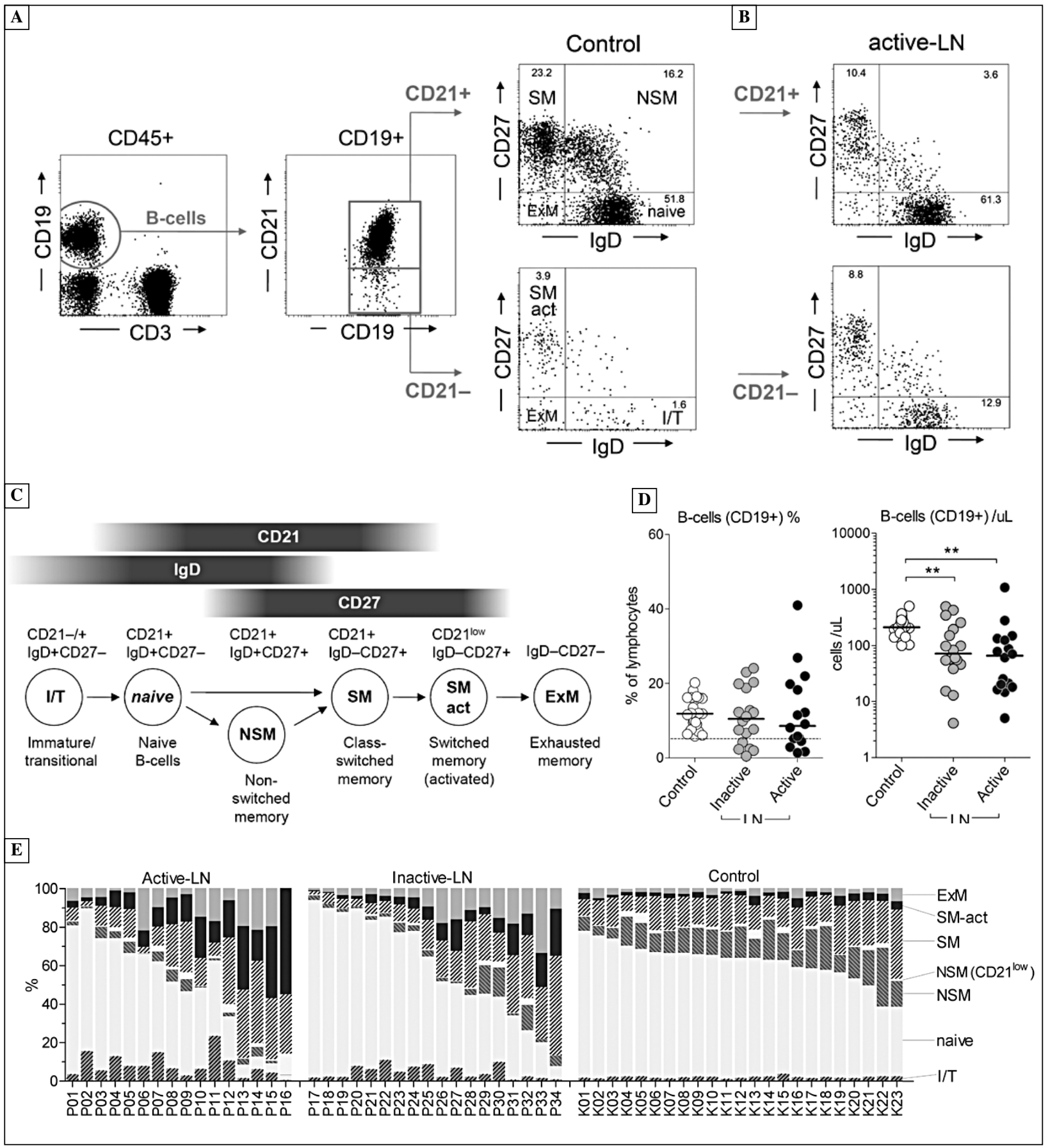

Figure 1. Subsets of memory B-cells in systemic lupus erythematosus (SLE) patients and control subjects. A. Flow cytometry (FC) gating strategy used to identify major subsets of CD19+ B-cells (details in the methods section, abbreviations explained in C). Dot-plots represent FC data of a control individual (K20); B. Similar gating strategy applied to FC data of lupus nephritis (LN) patient (P04); C. Diagram summarizing main subtypes of circulating CD19+ B-cells identified on the basis of the expression of CD21, CD27 and IgD; D. Percentage and counts of peripheral blood CD19+ B-cells in all studied groups. Horizontal bars represent medians; E. Percentages of different CD19+ B-cell subsets shown separately for each individual (data for CD21 ${ }^{\text {low }}$ NSM cells were also included)

$(R=0.48)$, dsDNA $(R=0.61)$ and complement components (e.g. $\mathrm{C} 4, R=-0.48)$. Additionally, percentage of activated SM B-cells showed weak association with SLEDAI score $(R=0.37)$ and peripheral blood lymphocyte count $(R=-0.35)$. As expected, absolute counts of naïve, NSM and resting SM B-cells also correlated with clinical markers of the disease (not shown). Interestingly, activated SM B-cells (but not 


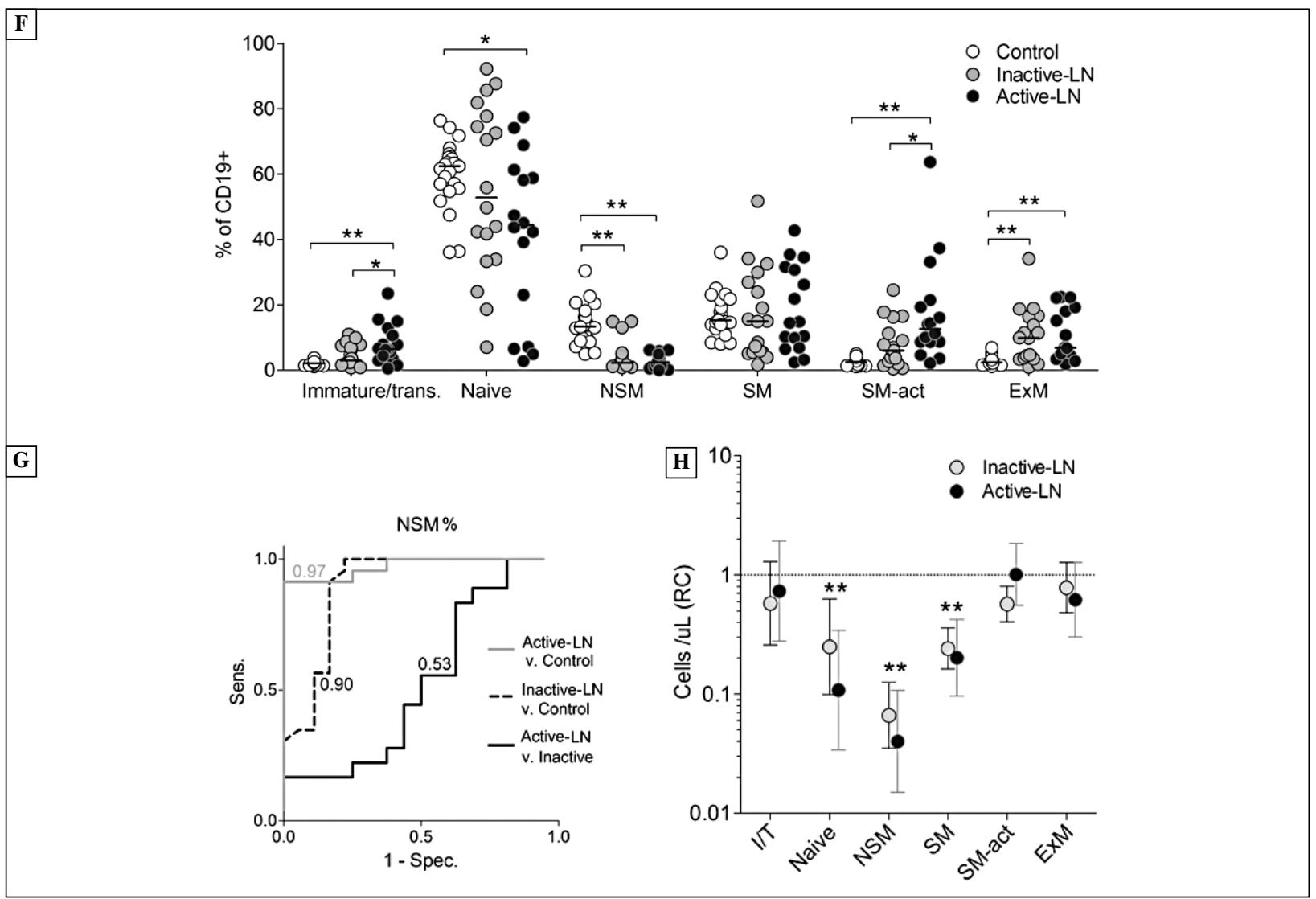

Figure 1. (cont.) Subsets of memory B-cells in SLE patients and control subjects. F. Differences in the percentage values of B-cells between all studied groups. Horizontal bars represent medians. ${ }^{*} P<0.05,{ }^{* *} P<0.01$; G. ROC curves of the prediction of a given disease phenotype based on NSM (\% of CD19+) data. Individual AUC (area under the curve) values are plotted next to each representative data-set; $\mathbf{H}$. Fold changes in the number of analyzed B-cell subsets (RC - relative to control group, e.g. value 0.1 means 10 -fold decrease, data presented as means and $95 \%$ CI). Naïve, NSM and resting-SM B-cells were significantly decreased $(* * P<0.01)$ in both active- and inactive-LN patients as compared with controls

other B-cell types) correlated weakly with daily urine protein excretion $(R=0.36)$ and urine sediment erythrocytes $(R=0.51)$. Percentage of memory B-cell subsets was comparable in LN-patients stratified based on renal biopsy status (data not shown).

\section{Increased percentage of effector memory \\ T-cells in $L N$}

The decrease in absolute number of T-cells was comparable in active- and inactive-LN (median 514 and 700 cells $/ \mu \mathrm{L}$, respectively, $v s .1388$ in control subjects, $P<0.01)$ and affected mostly CD4+ T-cells (237 and 409 cells $/ \mu \mathrm{L}$ in $\mathrm{LN}$ groups $v s .923$ in controls, $P<0.01$ ) resulting in lower percentage of CD4+ T-cell in $\mathrm{LN}$ (median $37 \%$ of total lymphocytes in all $\mathrm{LN}$ patients vs. $47 \%$ in controls) and decreased CD4/CD8 ratio (1.01 and 1.67 in active- and inactive-LN vs. 2.37 in controls, $P<0.01$ ). Additional staining for CD45RA, CD45RO and CCR7 (Figure 3A) allowed for identification of naïve and memory T-cell subsets (Figure 3B).
The main feature of T-cell compartment in $\mathrm{LN}$ was a significant decrease in the percentage of naïve T-helper cells $(36.8 \%$ in $\mathrm{LN}$ vs. $48.0 \%$ in control group, $P<0.05)$ and increase in the percentage of $\mathrm{T}_{\mathrm{EM}}$ subset (29.7\% vs. $14.5 \%$, respectively, $P<0.01$ ), with no difference between active and inactive disease (Figure 3C). The frequency of CD8+ T-cell subsets did not change considerably in LN (Figure 3C). Further analysis showed significant decrease in the absolute count of naïve and $\mathrm{T}_{\mathrm{CM}}$ cells (primarily $\mathrm{CD} 4+$ ) in $\mathrm{LN}$ as compared with control subjects, which was more prominent in the active disease (Figure 3D). Comparison of B-cell and T-cell memory fractions revealed a weak correlation of the percentage of CD4+ $T_{E M}$ with both NSM $(r=-0.42)$ and ExM B-cells $(r=0.48)$. Not surprisingly, there was a considerable correlation between the magnitude in the decrease of absolute counts of $\mathrm{CD} 4+\mathrm{T}$ and $\mathrm{B}$ fractions, which was most prominent in case of naïve CD4+ and NSM B-cells (Figure 3F). 




Figure 2. Association of B-cell subsets with clinical and laboratory parameters of lupus nephritis (LN). A. Percentage of $\mathrm{CD} 21^{\text {low }} \mathrm{B}$-cells in the studied groups. ${ }^{* *} P<0.01 ;$ B. Correlation matrix showing significant $(P<0.05$, bold $)$ associations between the main subsets of B-cells (abbreviations explained in Fig. 1C) and selected markers of active SLE (data from the whole SLE group). CRP - C-reactive protein, C3c - complement component 3Cc, C4 - complement component C4, PBL - peripheral blood lymphocytes (count), CTX - cyclophosphamide (cumulative dose); C. Scatter-plots showing the correlations of the percentage of immature/transitional B-cells with serum levels of C3c and SLEDAI score

\section{Discussion}

Earlier studies showed specific changes in B-cell compartment in SLE, though data on the association between various memory B-cells and disease activity are inconsistent $[18,28,29]$. To address this point we quantified different subsets of B-cells in patients with inactive $\mathrm{LN}$ and during disease exacerbations, and also checked if they are paralleled by similar changes within T-cell memory compartment. We defined main subsets of B-cells based on multicolor flow cytometry panel, including staining for CD27 (memory marker), surface-IgD and CD21 (complement receptor CR2, a component of BCR co-receptor complex), which allowed to distinguish switched and non-switched memory B-cells and additionally CD21 ${ }^{\text {low }}$ immature and activated B-cells.
Decreased frequency of NSM B-cells was the most prominent feature of LN in the current study. This alteration was observed in all $\mathrm{LN}$ patients with disease exacerbation and in a majority $(83 \%)$ of patients in remission. Our data is in line with previous reports showing deficiency in $\mathrm{CD} 27+\mathrm{IgD}+$ cells in SLE $[18,19]$, though in contrast to earlier study [18], we did not see any association of B-cell NSM percentage with disease activity status. The subset of NSM B-cells has been implicated in immune responses against T-cell independent antigens, however recent studies confirmed their role as a long-lasting memory to T-cell dependent antigens involved in reconstitution of switched memory pool upon antigen restimulation [10]. Changes in NSM fraction were accompanied by increased percentage of immature/ /early-transitional B-cells and activated SM cells, both 


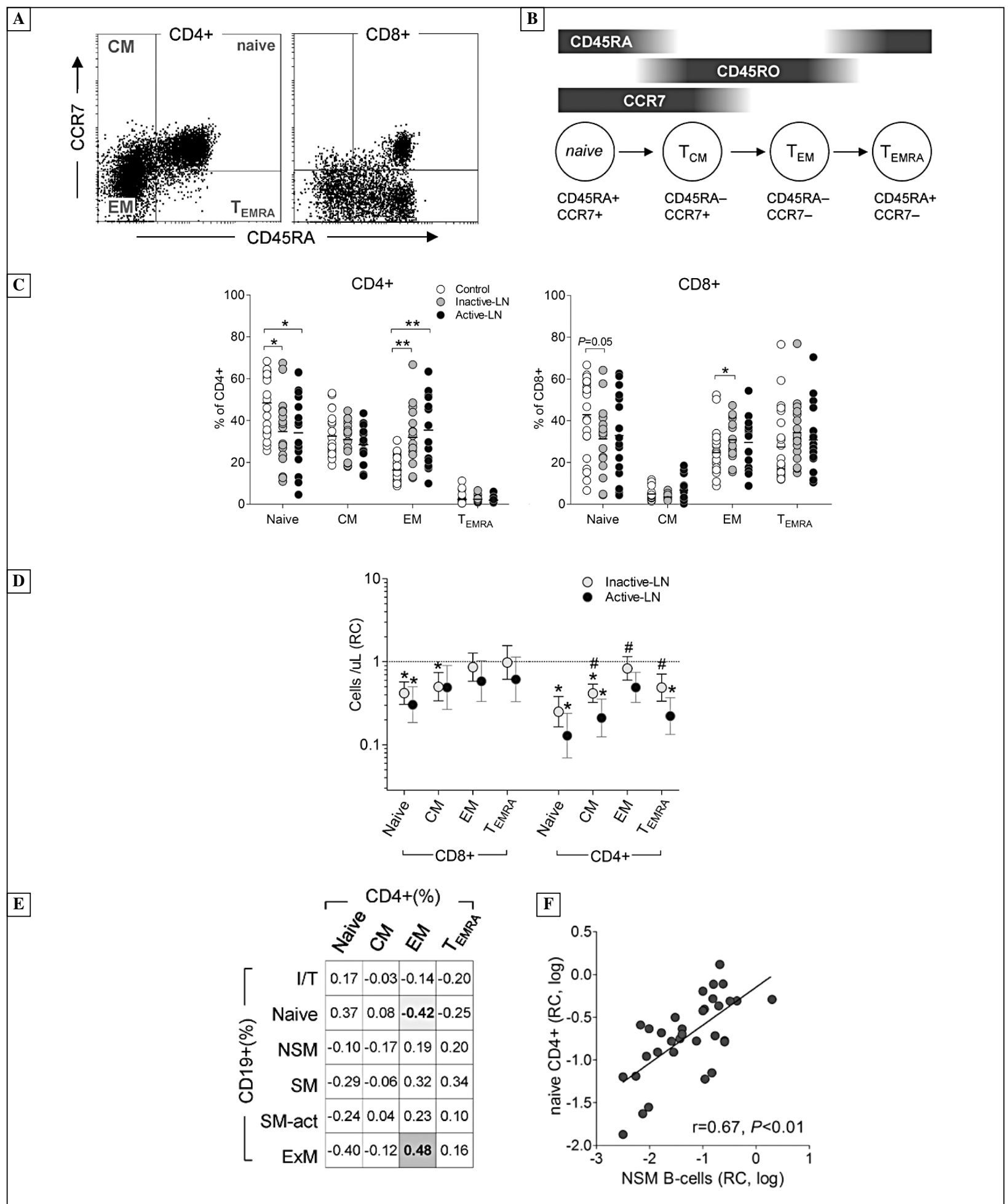

Figure 3. Memory T-cell subsets in systemic lupus erythematosus (SLE). A. Flow cytometry dot-plots (whole blood gated on CD4+ or CD8 + T-cells) showing major subsets of memory T-cells. CM - central memory, EM - effector memory, EMRA - effector memory CD45RA+; B. Simplified diagram showing the main stages of differentiation of memory T-cells and their candidate surface markers (based on $[16,22])$; C. Percentages (within CD4+ [left] or CD8+ [right] T-cells) of memory subsets in control subjects and lupus nephritis $(\mathrm{LN})$ patients. Horizontal bars represent mean values. ${ }^{*} P<0.05$, $* * P<0.01$; D. Graph showing the relative decrease in the absolute counts of CD8+ and CD4+ memory subsets in LN patients as compared with control group ( $\mathrm{RC}$ - relative to controls). Whiskers represent $95 \%$ CI. ${ }^{*} P<0.01$ in comparison with controls. ${ }^{\# P}<0.05$ between active and inactive $\mathrm{LN}$; E. Correlation matrix shows Pearson $\mathrm{r}$ coefficients in comparisons of major memory subsets of CD19+ B-cells (abbreviations explained in the Fig. 1C) and CD4+ T-cells in LN group. Significant $(P<0.05)$ associations are marked in bold font; F. Correlation between fold decrease (in comparison with mean value in the control group, $\mathrm{RC}$ - relative to control) in NSM B-cells and naïve CD4+ T-cells in LN patients 
subsets defined as CD21 $1^{\text {low }}$. During development of humoral response the loss of CD21 expression in CD27+ memory B-cells occurs during their activation and differentiation towards antibody secreting cells $[8,16,23]$. Nevertheless, systemic accumulation of $\mathrm{B}$-cells with $\mathrm{CD} 27+\mathrm{CD} 21^{\text {low }}$ phenotype, e.g. during chronic viral infections, is also considered as a sign of aberrant B-cell activation and dysfunction within the memory compartment $[21,30]$. In this study we observed very similar B-cell changes in $\mathrm{LN}$ patients. The fraction of activated SM B-cells $\left(\mathrm{CD} 27+\mathrm{CD} 21^{\text {low }}\right)$ was $\sim 3$-fold higher in patients with active $\mathrm{LN}$ as compared with control group. In line with previous reports $[17,18,29]$, we also observed increased frequency of double negative (i.e. CD27-IgD-) B-cells in patients with $\mathrm{LN}$. This fraction represents class-switched memory cells that lost CD27 expression, which are also markedly expanded during chronic infections, and resemble tissue-like exhausted memory B-cells $[23,32]$. These results suggest altogether that reduction in NSM B-cells is most likely a consequence of facilitated class switch of IgM+ memory cells and further differentiation towards SM-activated and exhausted memory phenotypes. This was further confirmed by analysis of absolute counts of B-cells. As it turned out, SLE related lymphopenia affected mostly naïve and NSM B-cells, but again, with no evident association with disease activity status. Moreover, despite clear increase in the percentage of immature/ /early-transitional cells, there was no change in their absolute count. This observation confirms that bone marrow production of B-cells is not disturbed in SLE, as has been suggested previously [33]. Instead, our results support the concept on preferential depletion of IgM+ memory pool in LN due to their constant activation (repeated exposure to self-antigens) and continuous replenishing of SM-pool. However other explanations, e.g. abnormal selection processes or diminished susceptibility of particular memory B-cells to immunosuppressive drugs, are still possible. But autoreactive B-cells are only a tiny fraction of all memory cells, so why the whole compartment is changed? Some hints came from recent study, showing that various neutrophil-derived proinflammatory mediators facilitate differentiation of $\mathrm{IgD}+$ CD27+ memory B-cells and IgG class switch [10]. Neutrophils participate in the pathogenesis of LN, and both neutrophil derived cytokines (e.g. CCL2) and markers of neutrophil activation (extracellular traps) correlate with the activity of glomerulonephritis and dsDNA titers [34, 35]. This implies that B-cell memory compartment in LN may also be shaped by innate immunity mediators released at the site of autoimmune inflammation. Because class switching and
B-cell activation are under strict control of T-helper cells we further explored if similar changes of immune memory developed also in T-cell compartment.

A characteristic feature of systemic autoimmunity is constant exposure to tissue antigens, which favors differentiation of long-lasting $\mathrm{T}_{\mathrm{EM}}$ cells with well-developed effector functions [12]. In SLE this phenomenon may be associated with impaired immunosuppression, due to persistently lower numbers of regulatory T-cells or their impaired function [36, 37]. Similarly to previous report [38], we observed lower frequency of naïve CD4+ T-cells and increased percentage of $T_{E M}$ fraction in both active- and inactive-LN. Additionally, we showed that SLE-related lymphopenia affected mostly naïve CD4+ lymphocytes. The reasons for decreased CD4+ lymphocyte count in SLE are, however, poorly understood. It may result from higher susceptibility of T-cells to apoptosis due to diminished expression of complement regulatory proteins or defective Fas/Fas-ligand system [39]. The depletion of lymphocytes may also be linked with anti-lymphocyte antibodies, which are detected in $\sim 50 \%$ of SLE patients presenting with lymphopenia [40]. Interestingly, we observed comparable decrease $(\sim 10$-fold) in absolute counts of antigen inexperienced T-cells and NSM B-cells. We also showed a correlation between the percentage of naïve fractions of B-cells and CD4 + T-cells, as well as between their mature subsets. This observation suggests that chronic antigenic stimulation and facilitated shift towards $T_{E M}$ may also contribute to lymphopenia in T-cell compartment. It is also tempting to speculate that decline in one compartment, e.g. in CD4+ T-cells, may trigger similar changes in the other. It has been shown that transient depletion of B cells can potently influence reactivation and maintenance of pathogen specific CD4+ T cells [40]. However, there are only scarce data on possible interaction between CD4+ lymphopenia and the concomitant quantitative changes in B cell subsets [23, 42].

The presented data highlight similarities in changes of B-cell and CD4+ T-cell subsets in LN patients, including deficiency in naïve (or non-switched) cells in all SLE patients and relative increase in effector memory (or exhausted) subsets during overt disease. LN-related changes in B-cell memory compartment, particularly the decline in NSM B-cells, may result from chronic antigenic stimulation and facilitated differentiation towards mature memory phenotypes, though it does not exclude possible intrinsic abnormalities related to abnormal selection processes or varying sensitivity to immunosuppressants. Marked decrease in the percentage of circulating $\mathrm{CD} 27+\mathrm{IgD}+$ memory B-cells was a typical signature of $\mathrm{LN}$, while 
the increase in $\mathrm{CD} 21^{\text {low }} \mathrm{B}$-cells could be used as additional laboratory marker of active disease. Whether B-cell memory subsets are skewed to the same extent in other SLE manifestations, or their diagnostic potential in SLE remains to be further studied.

\section{Acknowledgements}

This study was supported by National Science Center, Poland, grant number N402 627940.

\section{References}

1. Borchers AT, Leibushor N, Naguwa SM, Cheema GS, Shoenfeld Y, Gershwin ME. Lupus nephritis: a critical review. Autoimmun Rev. 2012;12:174-194. doi: 10.1016/j.autrev.2012.08.018.

2. Kaczmarczyk K, Kosalka J, Soja J, Kuzniewski M, Musial J, Okon K. Renal interstitial mast cell counts differ cross classes of proliferative lupus nephritis. Folia Histochem Cytobiol. 2014;52:218-224. doi: 10.5603/FHC.2014.0025.

3. Krishnan MR, Wang C, Marion TN. Anti-DNA autoantibodies initiate experimental lupus nephritis by binding directly to the glomerular basement membrane in mice. Kidney Int. 2012;82:184-192. doi: 10.1038/ki.2011.484.

4. Giles BM, Boackle SA. Linking complement and anti-dsDNA antibodies in the pathogenesis of systemic lupus erythematosus. Immunol Res. 2013;55:10-21. doi: 10.1007/ /s12026-012-8345-z.

5. Olson SW, Lee JJ, Prince LK et al. Elevated subclinical double-stranded DNA antibodies and future proliferative lupus nephritis. Clin J Am Soc Nephrol. 2013;8:1702-1708. doi: 10.2215/CJN.01910213.

6. Olsen NJ, Karp DR. Autoantibodies and SLE: the threshold for disease. Nat Rev Rheumatol. 2014;10:181-186. doi: 10.1038/nrrheum.2013.

7. Kurosaki T, Kometani K, Ise W. Memory B cells. Nat Rev Immunol. 2015;15:149-159. doi: 10.1038/nri3802.

8. Kaminski DA, Wei C, Qian Y, Rosenberg AF, Sanz I. Advances in human B cell phenotypic profiling. Front Immunol. 2012;3:302. doi: 10.3389/fimmu.2012.00302.

9. Pape KA, Taylor JJ, Maul RW, Gearhart PJ, Jenkins MK. Different B cell populations mediate early and late memory during an endogenous immune response. Science. 2011;331:1203-1207. doi: 10.1126/science.1201730.

10. Seifert M, Przekopowitz M, Taudien S et al. Functional capacities of human IgM memory B cells in early inflammatory responses and secondary germinal center reactions. Proc Natl Acad Sci USA. 2015;112:E546-E555. doi: 10.1073/ /pnas.1416276112.

11. Rekvig OP. The anti-DNA antibody: origin and impact, dogmas and controversies. Nat Rev Rheumatol. 2015;11:530-540. doi: 10.1038/nrrheum.2015.69.

12. Devarajan P, Chen Z. Autoimmune effector memory T cells: the bad and the good. Immunol Res. 2013;57:12-22. doi: 10.1007/s12026-013-8448-1.

13. Bhargava P, Calabresi PA. Novel therapies for memory cells in autoimmune diseases. Clin Exp Rheum. 2015;180:353-360. doi: $10.1111 /$ cei.12602.

14. Odendahl M, Jacobi A, Hansen A et al. Disturbed peripheral B lymphocyte homeostasis in systemic lupus erythematosus. J Immunol. 2000;165:5970-5979. doi: 10.4049/jimmunol.165.10.5970.

15. Dörner T, Jacobi AM, Lee J, Lipsky PE. Abnormalities of B cell subsets in patients with systemic lupus erythemato- sus. J Immunol Methods. 2011;363:187-197. doi: 10.1016/j. jim.2010.06.00.

16. Wei C, Jenks S, Sanz I. Polychromatic flow cytometry in evaluating rheumatic disease patients. Arthritis Res Ther. 2015;17:46. doi: 10.1186/s13075-015-0561-1.

17. Wei C, Anolik J, Cappione A et al. A new population of cells lacking expression of CD27 represents a notable component of the B cell memory compartment in systemic lupus erythematosus. J Immunol. 2007;178:6624-6633. doi: 10.4049/ jimmunol.178.10.6624.

18. Rodríguez-Bayona B, Ramos-Amaya A, Pérez-Venegas JJ, Rodríguez C, Brieva JA. Decreased frequency and activated phenotype of blood CD27 IgD IgM B lymphocytes is a permanent abnormality in systemic lupus erythematosus patients. Arthritis Res Ther. 2010;12:R108. doi: 10.1186/ar3042.

19. Wehr C, Eibel H, Masilamani M et al. A new CD21low B cell population in the peripheral blood of patients with SLE. Clin Immunol.2004;113:161-171. doi: 10.1016/j.clim.2004.05.010.

20. Korganow AS, Knapp AM, Nehme-Schuster H et al. Peripheral B cell abnormalities in patients with systemic lupus erythematosus in quiescent phase: decreased memory B cells and membrane CD19 expression. J Autoimmun. 2010;34:426-434. doi: 10.1016/j.jaut.2009.11.002.

21. Hochberg MC. Updating the American College of Rheumatology revised criteria for the classification of systemic lupus erythematosus. Arthritis Rheum. 1997;40:1725. doi: 10.1002/ /art.1780400928.

22. Weening JJ, D'Agati VD, Schwartz MM et al. The classification of glomerulonephritis in systemic lupus erythematosus revisited.J Am Soc Nephrol. 2004;15:241-250. doi: 10.1097/01. ASN.0000108969.21691.5D.

23. Cameron JS. Lupus nephritis. J Am Soc Nephrol. 1999;10:413-424. doi: 1046-6673/1002-0413\$03.00/0.

24. Moir S, Fauci AS. B cells in HIV infection and disease. Nat Rev Immunol. 2009;9:235-245. doi: 10.1038/nri2524.

25. Suryani S, Fulcher DA, Santner-Nanan B et al. Differential expression of CD21 identifies developmentally and functionally distinct subsets of human transitional B cells. Blood. 2010;115:519-529. doi: 10.1182/blood-2009-07-234799.

26. Farber DL, Yudanin NA, Restifo NP. Human memory T cells: generation, compartmentalization and homeostasis. Nat Rev Immunol. 2014;14:24-35. doi: 10.1038/nri3567.

27. Koch S, Larbi A, Derhovanessian E, Ozcelik D, Naumova E, Pawelec G. Multiparameter flow cytometric analysis of CD4 and CD8 T cell subsets in young and old people. Immun Ageing. 2008;5:6. doi: 10.1186/1742-4933-5-6.

28. Odendahl M, Keitzer R, Wahn U et al. Perturbations of peripheral B lymphocyte homoeostasis in children with systemic lupus erythematosus. Ann Rheum Dis. 2013;62:851-858. doi: 10.1136/ard.62.9.851.

29. Jacobi AM, Reiter K, Mackay M et al. Activated memory B cell subsets correlate with disease activity in systemic lupus erythematosus: delineation by expression of CD27, IgD, and CD95. Arthritis Rheum. 2008;58:1762-1773. doi: 10.1002/ /art.23498.

30. Sciaranghella G, Tong N, Mahan AE, Suscovich TJ, Alter G. Decoupling activation and exhaustion of B cells in spontaneous controllers of HIV infection. AIDS. 2013;27:175-180. doi: 10.1097/QAD.0b013e32835bd1f0.

31. Doi H, Tanoue S, Kaplan DE. Peripheral CD27-CD21-B-cells represent an exhausted lymphocyte population in hepatitis C cirrhosis. Clin Immunol. 2014;150:184-191. doi: 10.1016/j.clim.2013.12.001.

32. Fecteau JF, Côté G, Néron S. A new memory CD27-IgG+ B cell population in peripheral blood expressing $\mathrm{VH}$ genes 
with low frequency of somatic mutation. J Immunol. 2006;177:3728-3736. doi: 10.4049/jimmunol.177.6.3728.

33. Sims GP, Ettinger R, Shirota Y, Yarboro CH, Illei GG, Lipsky PE. Identification and characterization of circulating human transitional B. Blood. 2005;105:4390-4398. doi: 10.1182/blood-2004-11-4284.

34. Kaplan MJ. Neutrophils in the pathogenesis and manifestations of SLE. Nat Rev Rheumatol. 2011;7:691-699. doi: 10.1038/nrrheum.2011.132.

35. Villanueva E, Yalavarthi S, Berthier CC et al. Netting neutrophils induce endothelial damage, infiltrate tissues, and expose immunostimulatory molecules in systemic lupus erythematosus. J Immunol. 2011;187:538-552. doi: 10.4049/ /jimmunol.1100450.

36. Gómez-Martín D, Díaz-Zamudio M, Vanoye G, Crispín JC, Alcocer-Varela J. Quantitative and functional profiles of CD4+ lymphocyte subsets in systemic lupus erythematosus patients with lymphopenia. Clin Exp Immunol. 2011;164:17-25. doi: 10.1111/j.1365-2249.2010.04309.x.

37. Kleczynska W, Jakiela B, Plutecka H, Milewski M, Sanak M, Musial J. Imbalance between Th17 and regulatory T-cells in systemic lupus erythematosus. Folia Histochem Cytobiol. 2011;49:646-653. doi: 10.5603/FHC.2011.0088.

38. Fritsch RD, Shen X, Illei GG et al. Abnormal differentiation of memory $\mathrm{T}$ cells in systemic lupus erythematosus. Arthritis Rheum. 2006;54:2184-2197. doi: 10.1002/art.21943.

39. Amasaki Y, Kobayashi S, Takeda T et al. Up-regulated expression of Fas antigen (CD95) by peripheral naive and memory $\mathrm{T}$ cell subsets in patients with systemic lupus erythematosus (SLE): a possible mechanism for lymphopenia. Clin Exp Immunol. 1995;99:245-250. doi: 10.1111/j.1365-2249.1995.tb05540.x.

40. Li C, Mu R, Lu XY, He J, Jia RL, Li ZG. Antilymphocyte Antibodies in Systemic Lupus Erythematosus: Association with Disease Activity and Lymphopenia. J Immunol Res. 2014;2014:672126. doi: 10.1155/2014/672126.

41. Lund FE, Randall TD. Effector and regulatory B cells: modulators of CD4+ T cell immunity. Nat Rev Immunol. 2010;10:236-247. doi: 10.1038/nri2729.

42. Malaspina A, Moir S, Chaitt DG et al. Idiopathic CD4+ $\mathrm{T}$ lymphocytopenia is associated with increases in immature/transitional B cells and serum levels of IL-7. Blood. 2007;109:2086-2088. doi: 10.1182/blood-2006-06-031385.

Submitted: 14 September, 2015 Accepted after reviews: 5 April, 2016 Available as AoP: 14 April, 2016 